\title{
Scale effects on the performance of sawtooth spoilers in transonic rectangular cavity flow
}

\author{
A. J. Saddington ${ }^{1} \mathbb{D} \cdot$ K. Knowles $^{1} \cdot$ V. Thangamani $^{2}$
}

Received: 24 July 2015 / Revised: 2 October 2015 / Accepted: 18 November 2015 / Published online: 11 December 2015

(C) The Author(s) 2015. This article is published with open access at Springerlink.com

\begin{abstract}
An experimental study was conducted on the effectiveness of sawtooth spoilers at suppressing acoustic tones within a rectangular cavity with a length-to-depth ratio of five and a width-to-depth ratio of two, operating at a freestream Mach number of 0.71 . Whereas previous research has focussed on the ratio of spoiler height to boundary-layer thickness $(h / \delta)$, this study also considers the effect of the ratio of cavity length to boundary-layer thickness $(L / \delta)$. Using a combination of unsteady pressure measurements and particle image velocimetry, it was established that consideration of the magnitude of both parameters is important when designing passive control methods for transonic cavities. A correlation was developed which suggests that in order to suppress fully the cavity tones, a critical spoiler height, $h_{\mathrm{cr}}$, is defined such that $h_{\mathrm{cr}} / \delta=0.065 \leq L / \delta \leq 0.082$.

\section{List of symbols}

$\begin{array}{ll}D & \text { Cavity depth }(\mathrm{m}) \\ f & \text { Frequency }(\mathrm{Hz}) \\ h & \text { Height of spoiler }(\mathrm{m}) \\ K & \begin{array}{l}\text { Ratio of disturbance velocity in the shear layer to } \\ \text { the freestream velocity }\end{array} \\ L & \text { Cavity length (m) } \\ M & \text { Mach number } \\ n & \text { Acoustic mode number }\end{array}$
\end{abstract}

A. J. Saddington

a.j.saddington@cranfield.ac.uk

1 Aeromechanical Systems Group, Defence Academy of the United Kingdom, Cranfield University, Shrivenham, Swindon SN6 8LA, UK

2 Frigesco Ltd, Innovation Centre, University of Exeter, Exeter, Devon EX4 4RN, UK $u_{i} \quad$ Velocity vector components in Cartesian coordinates $\left(u_{1}, u_{2}, u_{3}\right) \equiv(u, v, w)$

$W \quad$ Cavity width (m)

$x \quad$ Streamwise distance from the leading edge of the cavity $(\mathrm{m})$

$y \quad$ Distance from the cavity exit plane normal to the floor (m)

$z \quad$ Spanwise distance from the cavity centre line (m)

$\delta \quad$ Boundary-layer thickness (to $\left.0.99 u_{\infty}\right)(\mathrm{m})$

$\gamma \quad$ Ratio of specific heats

OASPL Overall sound pressure level (dB)

SPL Sound pressure level (dB)

SWS Sawtooth spoiler

()$_{\infty} \quad$ Freestream value

() critical value

() mean Mean value

()rms Root-mean-square value

\section{Introduction}

Cavities are increasingly appearing in aerospace applications, such as weapons bays on high-speed aircraft. When the bay doors are open, the resulting cavity flow can produce high acoustic and aerodynamic loads that may lead to structural failure. The control of cavity flow oscillations is, therefore, of practical significance and has, consequently, been the subject of numerous studies (Shaw et al. 1988; Perng and Dolling 2001; Ukeiley et al. 2004; MacManus and Doran 2008; Lawson and Barakos 2009; Vikramaditya and Kurian 2009a, b; Lada and Kontis 2011; Martinez et al. 2012). The flow control method can be passive or active depending on the external energy input (Cattafesta et al. 2008). Active control, such as blowing (Lusk et al. 2012), has the advantage 
that closed-loop feedback can be included to adapt to different frequencies. On the other hand, passive control methods involve simple geometric modifications and do not involve any direct energy input (although energy may be extracted from the flow or may be needed to deploy a passive device).

The characteristic frequencies associated with cavity oscillations were first predicted by Rossiter (1966), but now are generally predicted using the modified Rossiter equation (Eq. 1) proposed by Heller et al. (1971), which accounts for the higher speed of sound within the cavity at high freestream Mach numbers.

$f_{n}=\frac{u_{\infty}}{L}\left(\frac{n-\alpha}{M_{\infty}\left[1+\frac{(\gamma-1)}{2} M_{\infty}^{2}\right]^{-\frac{1}{2}}+\frac{1}{K}}\right)$

where $f_{n}$ is the frequency corresponding to the $n$th mode, $K$ is the ratio of disturbance velocity in the shear layer to the freestream velocity (generally taken as 0.57 ), and $\alpha$ is an empirical constant employed to account for the phase lag between the passage of a vortical disturbance past the cavity trailing edge and the formation of an upstream travelling disturbance. The value of $\alpha$ depends on $L / D$ and is evaluated as: $\alpha=0.062(L / D)$. The ratio of specific heats, $\gamma$, is assumed to be equal to 1.4 for air.

Previous research has shown that the geometrical scale of the cavity has a decisive influence on the noise levels and flow type of a cavity (Thangamani et al. 2014). The relevance of the scale of a model can be seen in two different aspects. First, at a given Mach number, cavities can be exposed to different thicknesses of the incoming boundary layer depending on factors like the position of the weapons bay on the aircraft and the altitude at which the aircraft is flying. Secondly, it determines the factors to be considered when a control technique that has been tested in a wind tunnel only at sub-scale is implemented at full-scale. Scaling effects have been recognised as contributory factors when anomalies are identified in cavity flow data. Shaw (1989) and Ross (2001) in their experiments with different cavity scales and a fixed boundary-layer thickness attributed the cause of discrepancies in their observations to the variation in boundary-layer thickness with respect to the cavity length.

The aim of this article is to identify the capability of a sawtooth spoiler to suppress cavity acoustic tones under different scale conditions. The two scaling parameters considered are the ratio of the spoiler height to boundary-layer thickness, $h / \delta$, and the cavity length to boundary-layer thickness, $L / \delta$.

\section{Experimental set-up}

This section details the wind tunnel facility and test rig, including a description of the cavity models and the sawtooth spoilers. The experiments were conducted in a transonic wind tunnel with the cavity models attached to the side wall of the test section.

\subsection{Transonic wind tunnel}

The experimental studies were carried out in the Cranfield University transonic wind tunnel facility, located at the Defence Academy of the United Kingdom in Shrivenham. The wind tunnel is an ejector-driven, closed-circuit design with an atmospheric blow-off box to ensure that the stagnation pressure remains constant. The tunnel circuit is in the vertical plane, and a general layout is shown in Fig. 1. The wind tunnel has a working section that is $500 \mathrm{~mm}$ long, $206 \mathrm{~mm}$ wide and $228 \mathrm{~mm}$ high. Using a Compair L11010 , air is compressed, dried and stored at $724 \mathrm{kPa}$ (gauge) in a $34 \mathrm{~m}^{3}$ reservoir that supplies the air to the wind tunnel. The tunnel has a run time of approximately $8 \mathrm{~s}$ for a computer-controlled blowing pressure of approximately $500 \mathrm{kPa}$ (gauge) at a tunnel stagnation pressure of $102 \mathrm{kPa}$. Tests were conducted at freestream Mach numbers of 0.71 and 0.85 with mean tunnel working section static pressures of 73.1 and $63.5 \mathrm{kPa}$, respectively. The unit Reynolds numbers were calculated to be $12.3 \times 10^{6}$ and $13.1 \times 10^{6} \mathrm{~m}^{-1}$, respectively. The uncertainty in the centre-line Mach number was obtained from five tunnel runs of 65,536 samples each and calculated at $95 \%$ confidence level as \pm 0.01 .

The natural boundary layer developed over the tunnel wall was used for the studies. The onset boundary-layer thickness, $\delta$, was estimated by measuring the stagnation pressure profile at a distance $45 \mathrm{~mm}$ upstream of the leading edge of the cavity model and was defined as the height from the wall to where $u / u_{\infty}=0.99$. The boundary-layer thickness was determined from the measured profile to be $8.0 \mathrm{~mm}$ (see Fig. 2).

\subsection{Cavity rig}

The scaling studies were undertaken using three rectangular cavity models, mounted into the tunnel side wall, whose corresponding linear dimensions were in the ratio 1:2:4. For convenience, these cavities are referred to as cavity A, cavity B and cavity $\mathrm{C}$, respectively. The cavity dimensions chosen were: $L \times W \times D=80 \mathrm{~mm} \times 40 \mathrm{~mm} \times 16 \mathrm{~mm}$ (cavity A); $160 \mathrm{~mm} \times 80 \mathrm{~mm} \times 32 \mathrm{~mm}$ (cavity B); and $320 \mathrm{~mm} \times 160 \mathrm{~mm} \times 64 \mathrm{~mm}$ (cavity C). The $L / D$ and $L / W$ ratios were, therefore, five and two, respectively, for all three cavities. The upstream edge of each cavity was maintained in the same streamwise position in the wind tunnel by inserting one of the three different cavity models (A, B or C) into the test rig (Fig. 3).

The sawtooth spoilers were composed of triangular elements as shown in Fig. 4. In order to maintain the same 


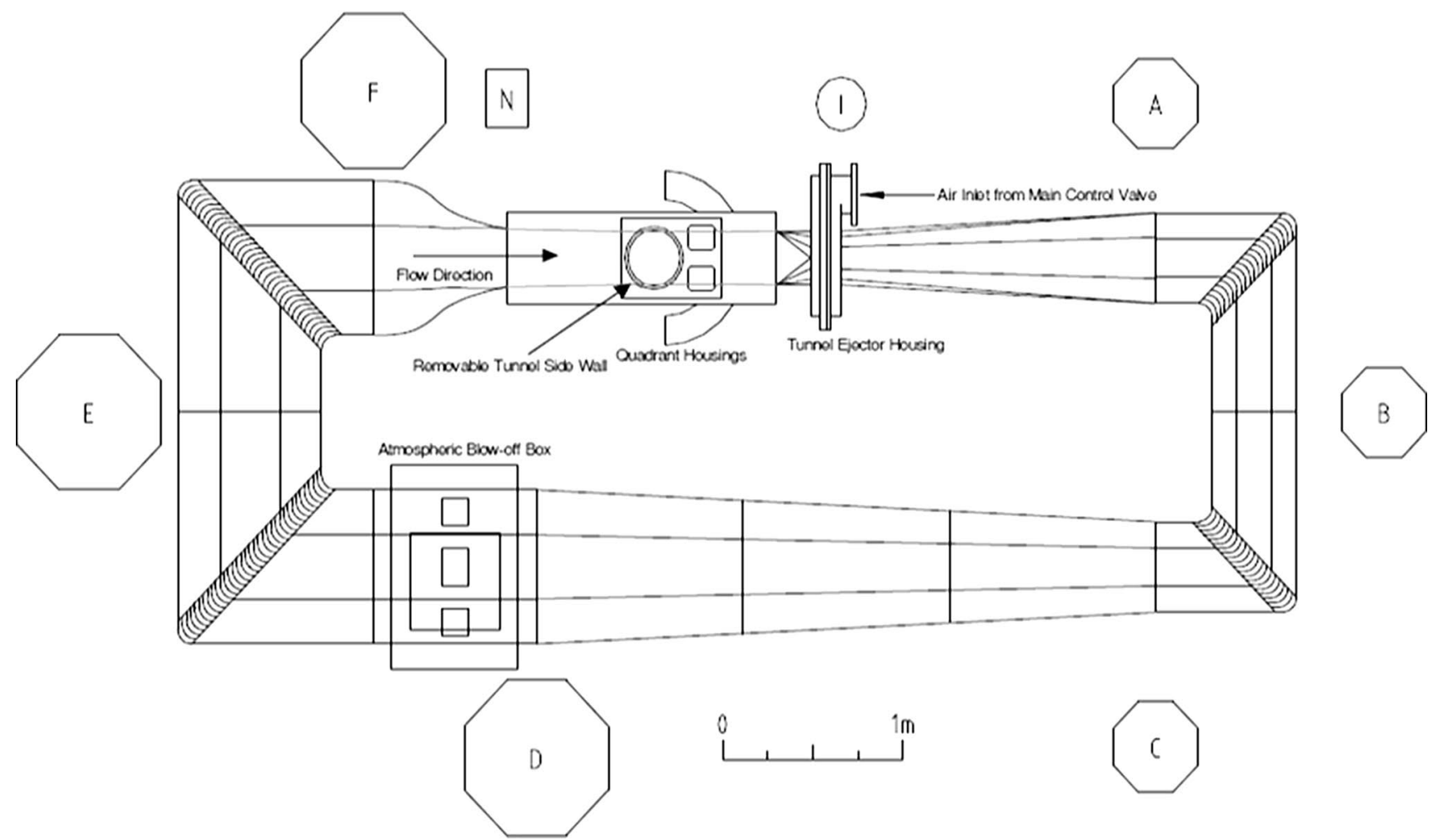

Fig. 1 Transonic wind tunnel layout: sections $A-F$ show the wind tunnel cross sections at various points; sections $N$ and $I$ are the nozzle and ejector planes, respectively

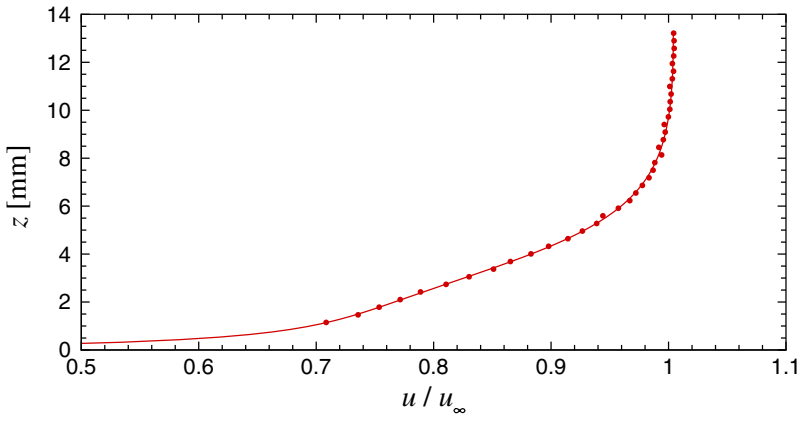

Fig. 2 Boundary-layer profile $45 \mathrm{~mm}$ upstream of the cavity leading edge

cavity $L / D$ for all tests, a $2-\mathrm{mm}$ pocket was machined into the front wall of each cavity to which the spoilers were fixed. Table 1 shows the different sawtooth spoiler geometries and the different cavities on which they have been tested. The reference axis system used for the experiments is shown in Fig. 4; the origin is at the centre of the leading edge of the cavity. The $x$-axis is parallel to the flow direction; the $y$-axis is normal to the cavity exit plane.

On the cavity floor, nine pressure tappings of $0.8 \mathrm{~mm}$ diameter were spaced linearly in the streamwise direction at increments of $x / L=0.1$. Three such rows were placed at planes $z / W=0, z / W=0.1625$ and $z / W=0.325$, which are referred to as "Centreplane" (CP), "Offsetplane1" (OP1) and "Offsetplane2" (OP2), respectively. In total, there were 27 static pressure tappings for the cavity model, forming a measurement grid on the floor as shown in Fig. 5. Flow visualisation of a cavity with the same $L / D$ and $L / W$ ratios by Atvars et al. (2009) indicated that the mean cavity flowfield is reasonably symmetric about the centreline. Hence, symmetry is assumed, and the pressure tappings have been made only for half of the floor plane.

\subsection{Instrumentation}

\subsubsection{Pressure measurements}

The unsteady pressure measurements in the experimental tests were made with an integrated pressure measurement device, the Scanivalve ZOC22B, and data were collected from the pressure tappings made on the cavity floor. The analogue signal from the ZOC22B was acquired by a modular amplifier system (DEWE-RACK) which has an analogue-to-digital converter. The signal was amplified, conditioned and digitised before being sent to a computer. A National Instruments DAQ card 6036E was used as the interface between the computer and the DEWE-RACK. 


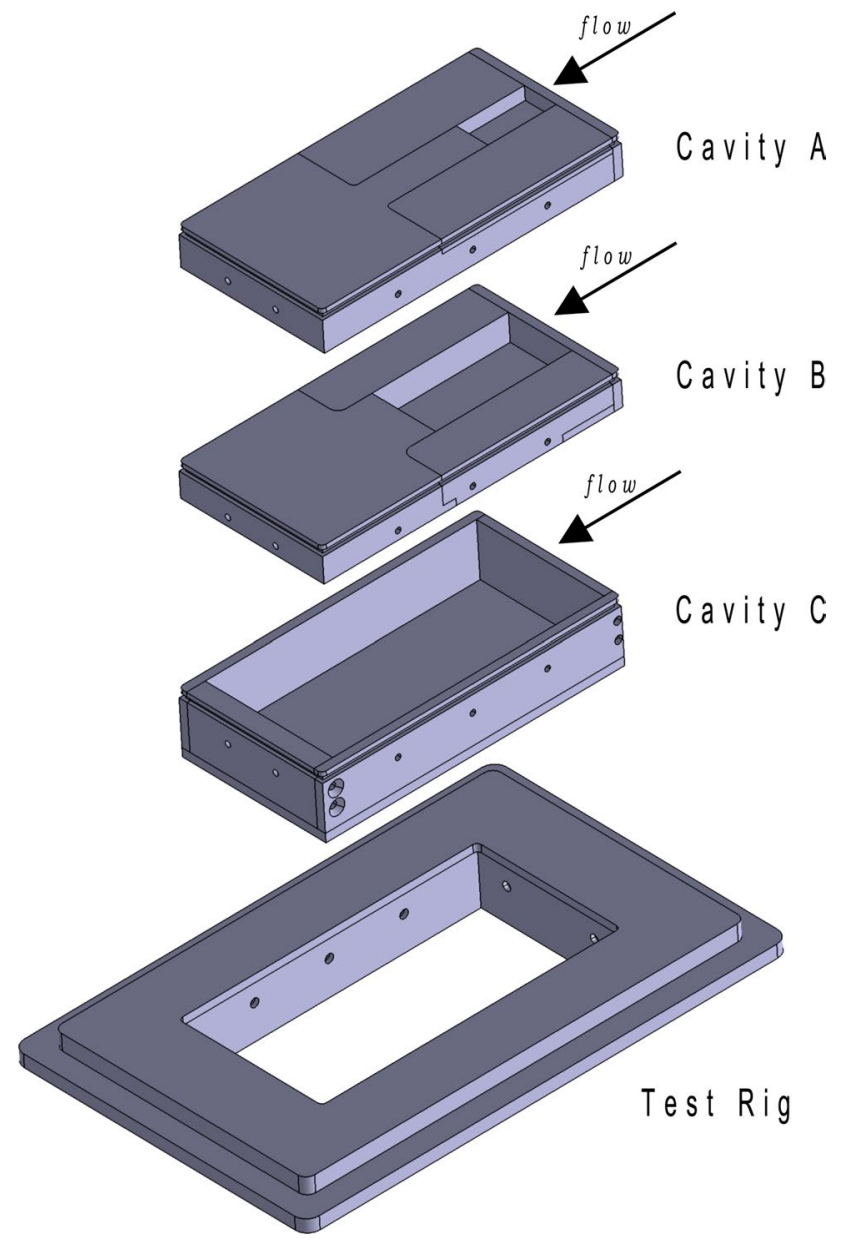

Fig. 3 Exploded view of the test rig with different cavity models

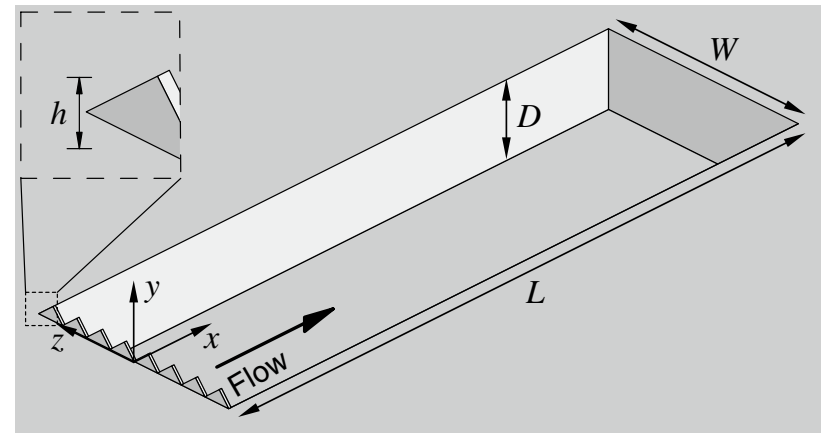

Fig. 4 General schematic of the cavity geometry and sawtooth spoiler passive control device: the spoiler pitch is defined as the distance between adjacent 'teeth'

For the spectral analysis of the unsteady pressure samples, a total of 65,536 samples, averaged over four tunnel runs, were recorded at a sampling rate of $12.5 \mathrm{kHz}$. The power spectra were plotted using an FFT algorithm to determine the amplitude of the different frequency components. The maximum error in amplitude of the tones was determined to be $0.208 \mathrm{~dB}$.
Table 1 Sawtooth spoiler geometries tested: pitch is defined as the distance between adjacent 'teeth'

\begin{tabular}{lccll}
\hline Total width $(\mathrm{mm})$ & Pitch $(\mathrm{mm})$ & $h(\mathrm{~mm})$ & $h / \delta$ & Cavity \\
\hline 40 & 5 & 2 & 0.25 & A \\
40 & 10 & 8 & 1 & A \\
40 & 10 & 16 & 2 & A \\
80 & 10 & 4 & 0.5 & B \\
80 & 10 & 8 & 1 & B \\
80 & 10 & 16 & 2 & B \\
160 & 10 & 8 & 1 & C \\
160 & 20 & 16 & 2 & C \\
160 & 20 & 24 & 3 & C \\
160 & 20 & 32 & 4 & C \\
\hline
\end{tabular}

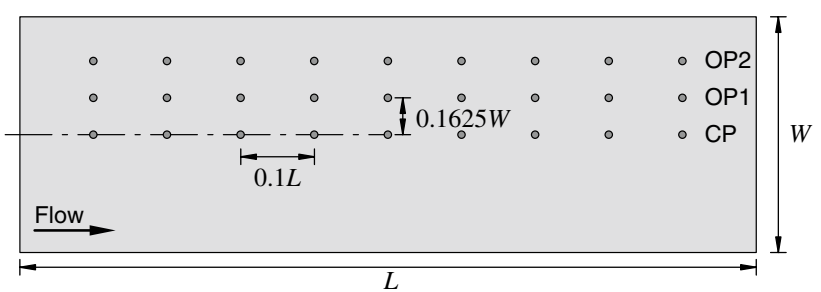

Fig. 5 The arrangement of pressure tappings on the cavity floor

\subsubsection{Velocity measurements}

Two-dimensional, time-averaged velocity field measurements were made using particle image velocimetry (PIV) on purpose-built, transparent cavities. The PIV equipment consisted of a New Wave Gemini II Nd:YAG double-pulsed laser which, through the use of a pair of spherical lenses, created a light sheet approximately $1 \mathrm{~mm}$ thick. Since the wind tunnel had no optical access at right angles to the laser sheet, the image was obtained using a front-coated mirror oriented at $45^{\circ}$ to the cavity side wall (Fig. 6). With the exception of the transparent floor and wall, all the sides of the cavity models and the test rig were painted black for the PIV experiments to prevent stray reflections of the laser light.

Water was used for seeding the flow. Filtered clean water was pressurised using a three-stage pump and sprayed through a rake of Bete atomising nozzles positioned across the span of the settling chamber of the wind tunnel. The rake consisted of three atomisers located at equal intervals at the mid-height position of the test section. The nominal seeding particle diameter based on a seeding pressure of 2000 psi is quoted by Ritchie (2005) as $10 \mu \mathrm{m}$.

All the PIV measurements given in this work were obtained from the mid-plane of the cavity $(z / W=0)$. The double-pulse frequency of the laser was set at $15 \mathrm{~Hz}$ with a pulse separation of $6 \mu \mathrm{s}$. The images were acquired 
Fig. 6 Schematic of the PIV set-up. a Top view. b Side view

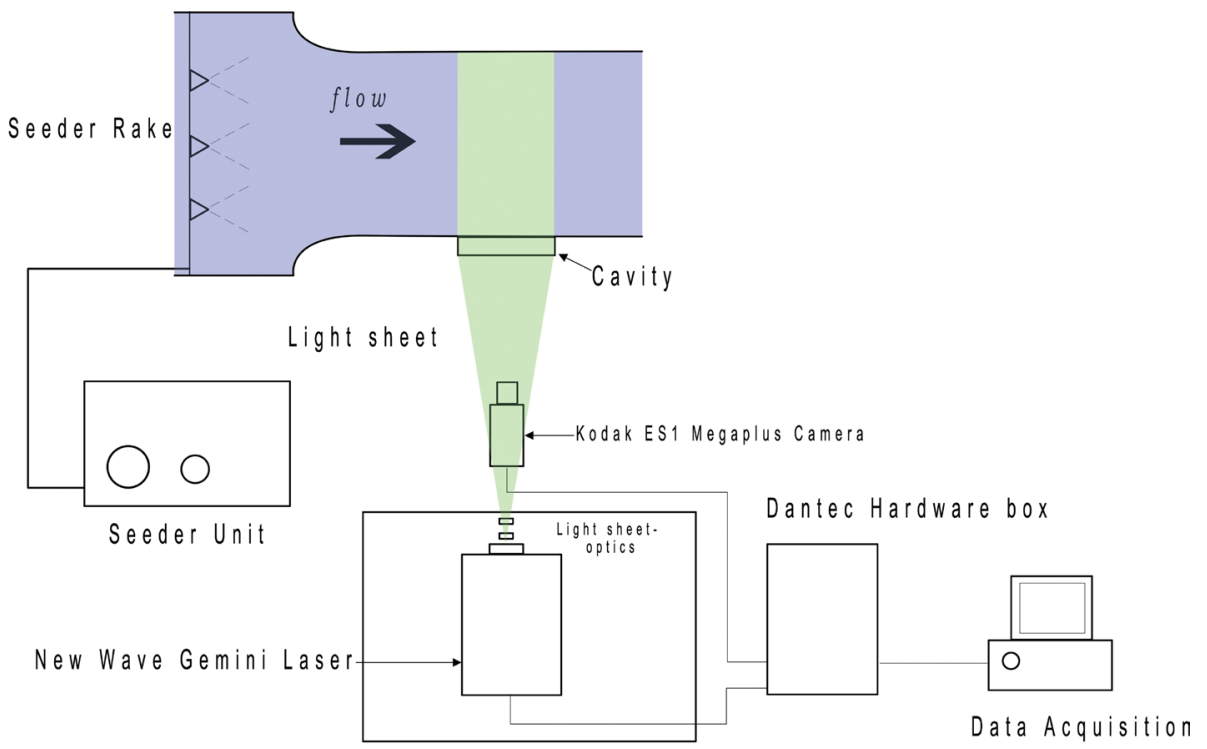

(a)

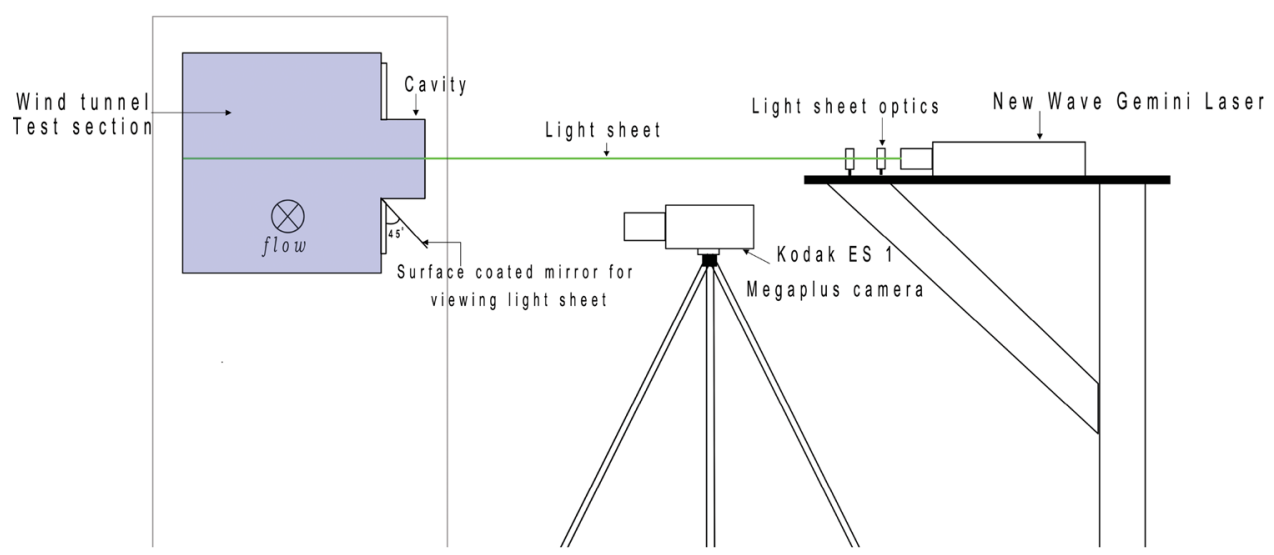

(b) using a $1016 \times 1008$ pixel resolution Kodak ES 1 Megaplus camera fitted with a Nikkor $60 \mathrm{~mm}$ f2.8 lens. A total of 700 image pairs acquired over 10 tunnel runs were used to create a single mean velocity plot. A Dantec Flow Map 500 acquisition controller was used as the hardware box, which worked in conjunction with Flow Manager (v3.21) software. Both the camera and laser were connected to the hardware box, and the timing and acquisition functions of both were controlled using the Flow Manager software. Due to the large size of the cavity model and limited resolution of the camera, the PIV data for cavity $\mathrm{C}$ were created from two separate sets of acquisitions. Image pairs for cavity segments $x / L=0$ to $x / L=0.5$ and $x / L=0.5$ to $x / L=1$ were acquired separately and combined together to get the final data for the whole length of the mid-plane. Post-processing of all PIV data was done using DaVis software from LaVision. A three-pass window deformation algorithm, with initial and final interrogation sizes of $32 \times 32$ pixels and $12 \times 12$ pixels and a $50 \%$ overlap between subsequent interrogation areas, was used to create the vectors. The time-averaged data were obtained from the ensemble average of 700 image pairs.

The uncertainties in PIV measurements stem from a number of factors which include errors occurring due to the particle flow dynamics, bias errors, random errors and statistical error in the mean and rms velocities, which are strongly related to the sample size. To remove outliers, the median filter proposed by Westerweel (1994) was used to locate spurious vectors and replace them by interpolation. On average, over $95 \%$ of the vectors in a given field were found to be valid prior to replacement and interpolation. The accuracy with which the position of the correlation peak is determined can be affected by bias errors. A source of bias error in PIV measurements is the velocity gradients 
present inside the interrogation area. This is because particles with higher velocities tend to leave the interrogation area between the pulses, biasing the result towards lower velocities. This has been minimised by selecting displacement limits between the pulses and by using window deformation algorithms as described above to increase the number of matched particles. Progressively decreasing the interrogation window size reduces the loss of particles from the interrogation area, leading to improved accuracy and better signal-to-noise ratios (Keane and Adrian 1992). Bias error can also occur due to the phenomenon of peak locking. Peak locking occurs because of the bias of the estimation of the position of the correlation peak towards an integer number of pixels. Peak locking is attributable mainly to the choice of the sub-pixel estimator used to locate the correlation peak. In the current experiments, a Gaussian subpixel estimator was used which was shown by Westerweel (1997) to be superior in terms of mitigating peak-locking effects. Random errors in PIV can arise from background noise, shot noise (which can vary from pixel to pixel) and random errors associated with properly identifying the sub-pixel displacement. Although these errors can play an important role in the determination of instantaneous PIV results, their effect on ensemble averages of large numbers of samples (as is the current case) can be considered negligible owing to the random nature of their occurrence. Therefore, random errors do not degrade the accuracy of statistics derived from larger PIV ensembles (Christensen 2004). Overall, the maximum error in the mean and RMS velocities was determined to be 2.8 and $2.6 \%$, respectively.

\section{Results and discussion}

The results for the experiments made at Mach 0.71 and 0.85 showed only minor differences, with the overall trends and observations being the same. As a consequence, only results for the Mach 0.71 tests will be presented here. The effectiveness of sawtooth spoilers across different scales was initially tested by using spoilers of the same profile and height, $h / \delta=1$. Spoilers with a height approximately equal to the boundary-layer thickness have been tested by a number of researchers including Rossiter (1966), Shaw et al. (1988), Ashworth (2008) and Lawson and Barakos (2009). Figure 7 shows the power spectra of cavities A, B and $\mathrm{C}$ with and without the use of sawtooth spoilers. The first three predicted Rossiter tones, R1, R2 and R3, calculated from Eq. 1, are also shown in the plots. It can clearly be observed that the effectiveness of the spoilers is not the same for all three cavities.

With the use of spoilers, there is a significant decrease in both the broadband and tonal amplitudes for cavities A and $\mathrm{B}$. Cavity A has a maximum reduction of $5 \mathrm{~dB}$ in its

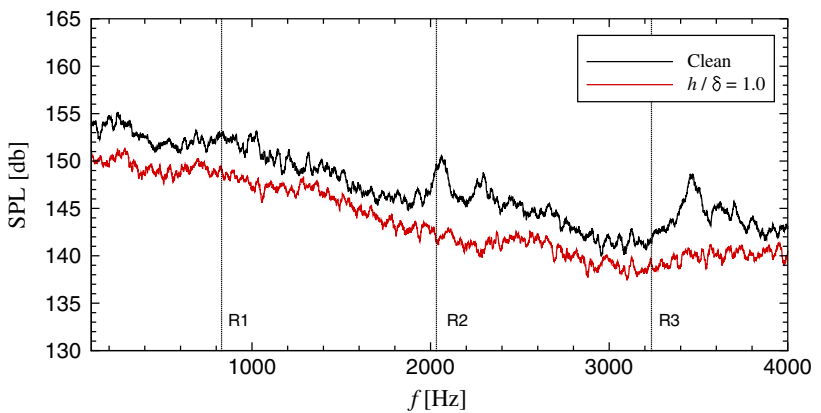

(a) Cavity A.

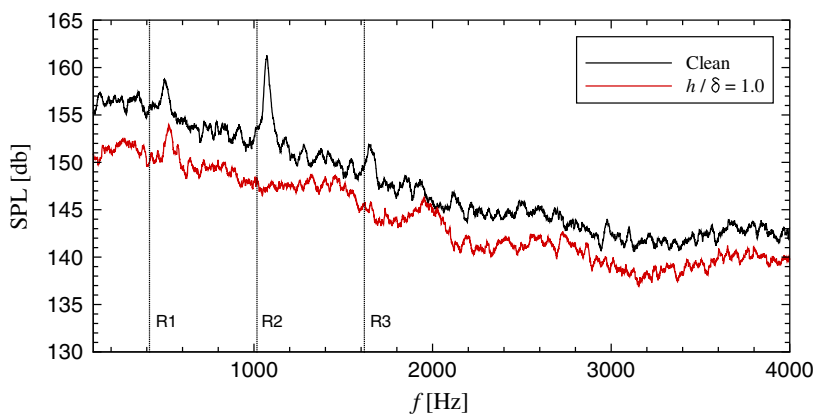

(b) Cavity B.

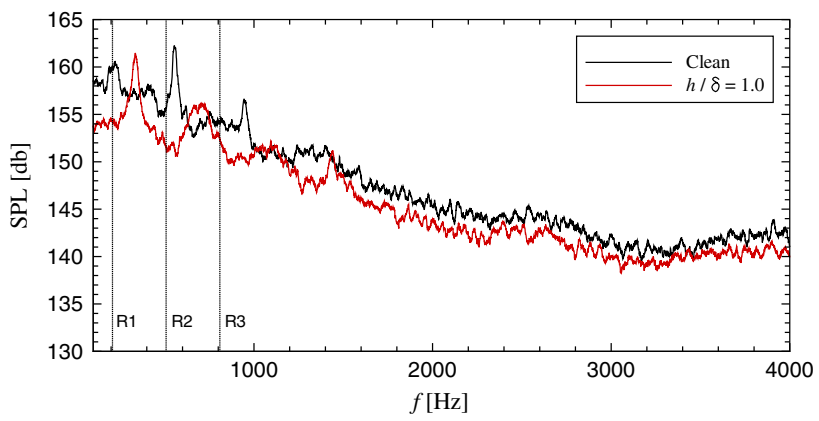

(c) Cavity C.

Fig. 7 The effect of sawtooth spoilers on the sound pressure levels for cavities A, B and C $(h / \delta=1.0, x / L=0.9)$

dominant tone (R1), and tones $\mathrm{R} 2$ and $\mathrm{R} 3$ are reduced to broadband levels. For cavity $\mathrm{B}$, there is a maximum reduction of $12.7 \mathrm{~dB}$ for its dominant tone (R2) which is no longer dominant. R1 is now the dominant tone, and R3 has been shifted to a higher frequency at around $2 \mathrm{kHz}$. The spoiler has very little effect on the amplitude of the tones for cavity C. Although there is a reduction of $7.4 \mathrm{~dB}$ in the dominant tone, the power spectrum is not reduced to broadband. There are high-amplitude peaks present even after the use of the spoiler, although with reduced magnitude when compared to the clean cavity. This suggests that the same spoiler is unable to cope with the increase in flow unsteadiness caused by the increase in cavity scale $(L / \delta)$.

To gain a better understanding of the effect that the spoilers were having on the flowfield, the PIV data for each 
Fig. 8 The effect of a sawtooth spoiler on the cavity streamwise velocity field of cavity A $(h / \delta=1)$

Fig. 9 The effect of a sawtooth spoiler on the cavity streamwise velocity field of cavity $B$ $(h / \delta=1)$

Fig. 10 The effect of a sawtooth spoiler on the cavity streamwise velocity field of cavity $\mathrm{C}(h / \delta=1)$
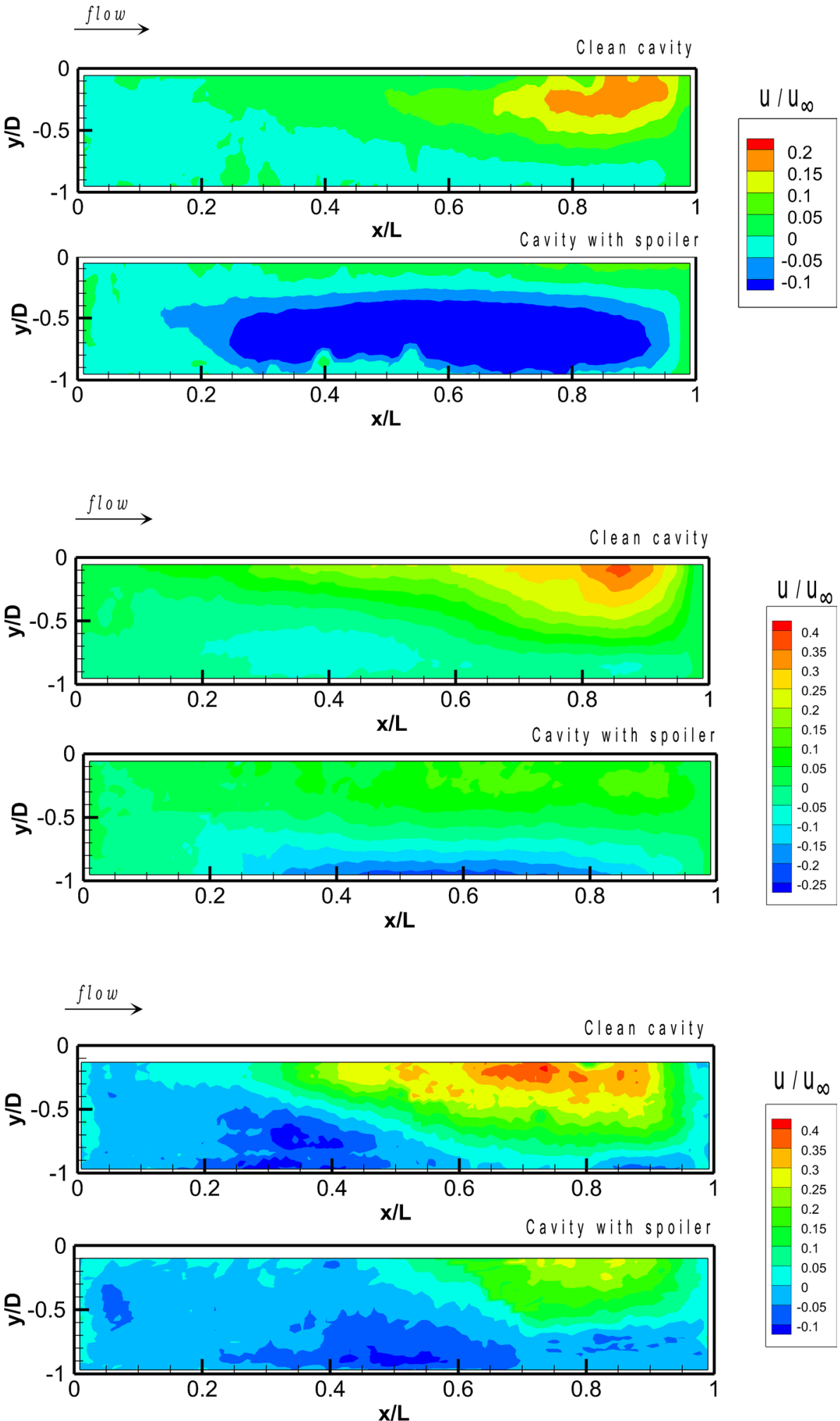

cavity have been compared. Figures 8,9 and 10 show that there is a significant change in the flowfield inside the cavity due to the presence of the spoilers. The velocity in the streamwise direction has been reduced significantly when compared to the clean cavity cases. This reduction in $u$ supports the belief that the spoilers deflect the shear layer away from the cavity. Due to the presence of the spoiler, the shear layer is vertically displaced or 'lifted', which decreases its interaction with the aft wall. This leads to a decreased momentum transfer to the acoustic source 
region, which is considered the main reason for tone amplification. It is worth noting, however, that lifting of the shear layer alone would not be responsible for the decrease in momentum transfer. Spoilers introduce significant threedimensionality into the shear layer and decrease its spatial coherence. They also thicken the shear layer due to vorticity induced in the streamwise direction. These factors also contribute to a reduced momentum transfer from the freestream to the vicinity of the aft wall.

The decreased momentum transfer and strength of the acoustic feedback result in tone attenuation that has been observed in the frequency spectra (Fig. 7). While the maximum values of $u_{\text {mean }}$ for cavities A, B and C were observed to be $18.1,36.1$ and $41 \%$ of the freestream velocity for the respective clean cavities, these maxima have reduced to $4.9,15.2$ and $28 \%$ for cavities with a spoiler. This indicates $73.2,57.7$ and $31.3 \%$ reduction in the values of peak streamwise velocity with a spoiler for cavities A, B and C, respectively. The velocity reduction is not constant, therefore, for all three cavities. The fluctuating vertical velocity, $v_{\text {rms }}$, also showed a non-constant magnitude relative to the freestream velocity, with larger-scale cavities showing higher vertical velocity components (Fig. 11). Due to the use of the spoilers with the same $h / \delta$, the length up to which the shear layer is vertically displaced should be equal. It is suggested that this is the reason for a stronger interaction between the shear layer and the cavity trailing edge as $L / \delta$ is increased.

Another significant change that occurs due to the spoilers is the increase in the mean values of negative streamwise velocity $(-u)$. The increase in negative velocities is due to the lifting of the shear layer and a decrease in pressure inside the cavity (Thangamani et al. 2014). The decrease in the positive streamwise velocity and increase in the negative streamwise velocity are not consistent across the different cavity scales as clearly shown in Fig. 12, which shows the percentage change in the values of maximum $+u$ and maximum $-u$ with the use of spoilers. Cavity $\mathrm{A}$ is most affected by the use of spoilers and cavity $\mathrm{C}$ the least affected. Where cavity tones are not fully suppressed, the change in negative streamwise velocities with the use of spoilers is likely to be responsible for the change in frequency of the tones observed in the power spectra due to a change in the velocity of the acoustic feedback wave.

Although spoilers with $h / \delta=1$ were able to attenuate totally the cavity tones and reduce the frequency spectrum to broadband level for small scales (cavity A), it was also found that the spoilers were incapable of suppressing cavity tones and unsteadiness at the larger scales (cavities B and C). This suggests that leading-edge spoilers cannot be scaled to the boundary-layer thickness alone to achieve suppression. When a change in $L / \delta$ results in a less-effective spoiler, the only practical solution is to increase its
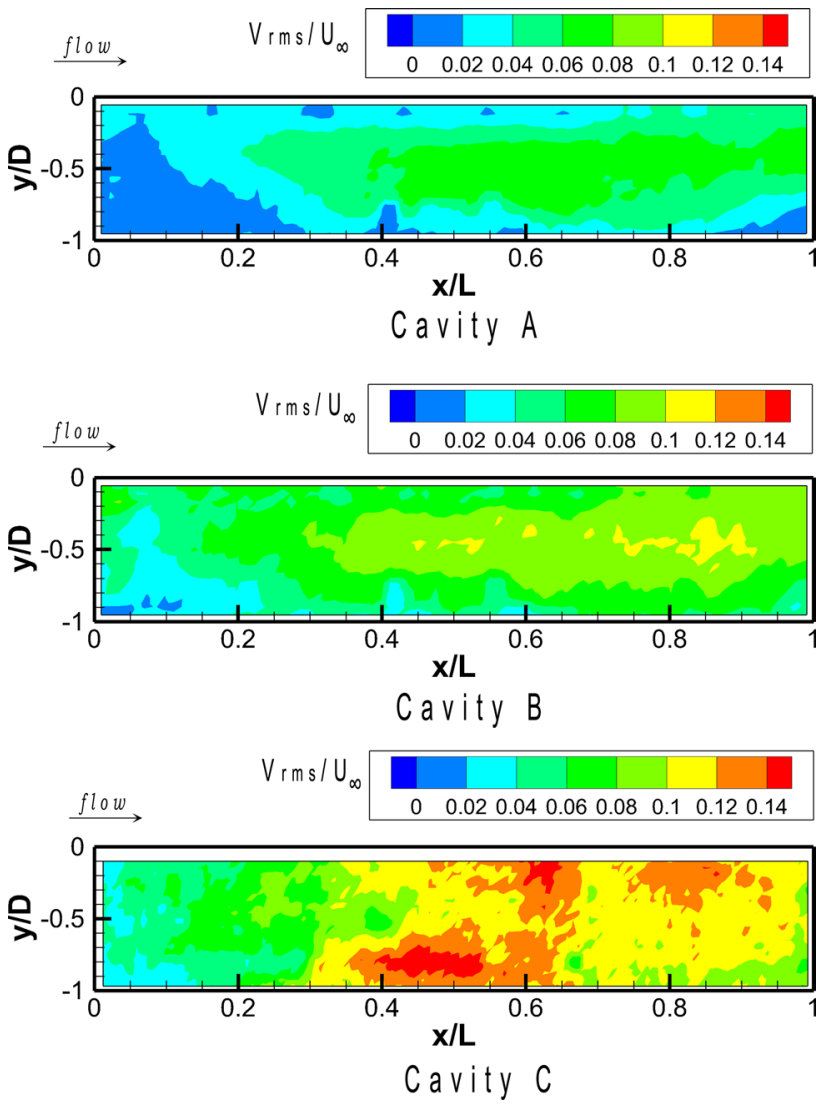

Fig. 11 Fluctuating vertical velocity, $v_{\text {rms }}$, for different cavities with sawtooth spoilers $(h / \delta=1)$

height, $h$. To deduce the correct spoiler height required for a particular value of $L / \delta$ requires the testing of different spoiler heights at different values of $L / \delta$.

Figure 13 shows the power spectra of the different cavities with various spoiler heights. It can clearly be seen that, in general, increasing the spoiler height, $h$, decreases the amplitude of the cavity tones and the broadband noise. It can also be seen from Fig. 13a that increasing the spoiler height from $h / \delta=0.25$ to 1 for cavity A decreases the tonal amplitudes and broadband noise significantly. However, increasing the spoiler height from $h / \delta=1$ to $h / \delta=2$ does not have much effect. This shows that the reduction in cavity noise reaches a saturation level at a particular spoiler height. This happens when all the cavity tones get reduced to a minimum broadband level achievable. The same behaviour can also be noticed for cavity $\mathrm{C}$ when $h / \delta$ is increased from three to four. Once the tones are attenuated to a broadband level, any further increase in spoiler height has no effect on noise reduction. The value of spoiler height at which this saturation is attained will be called the critical spoiler height, $h_{\mathrm{cr}}$. The value of OASPL reached after attaining the $h_{\mathrm{cr}}$ is not the same, however, for the different scales. This is because the OASPL obtained 
Fig. 12 Percentage change in streamwise velocity from clean cavity with the use of spoilers $(h / \delta=1)$
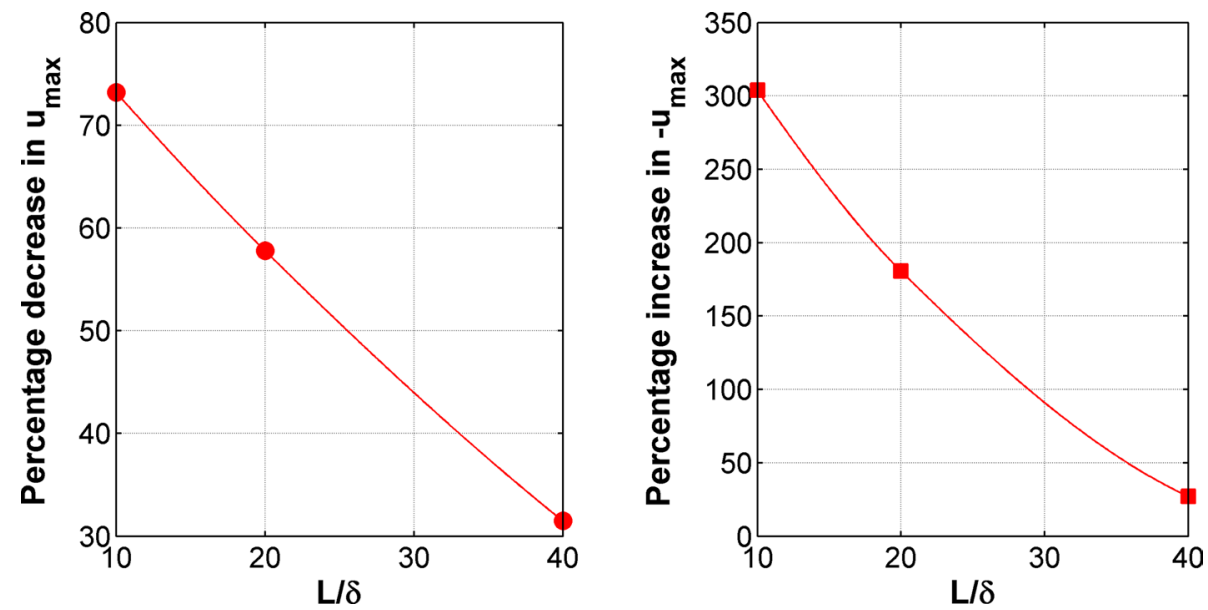

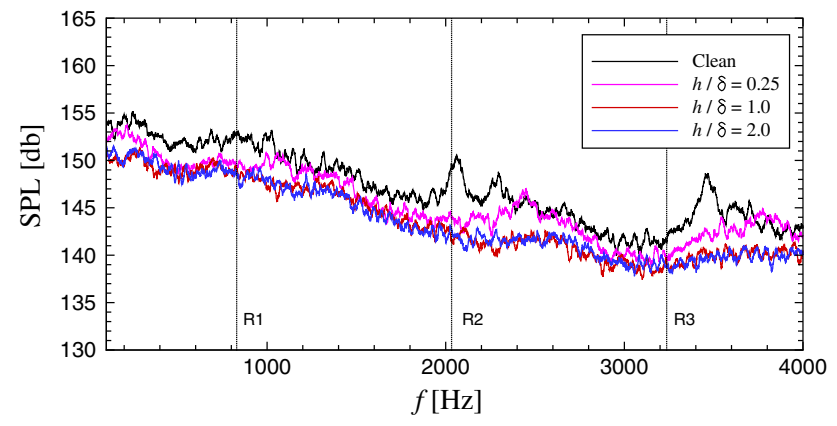

(a) Cavity A.

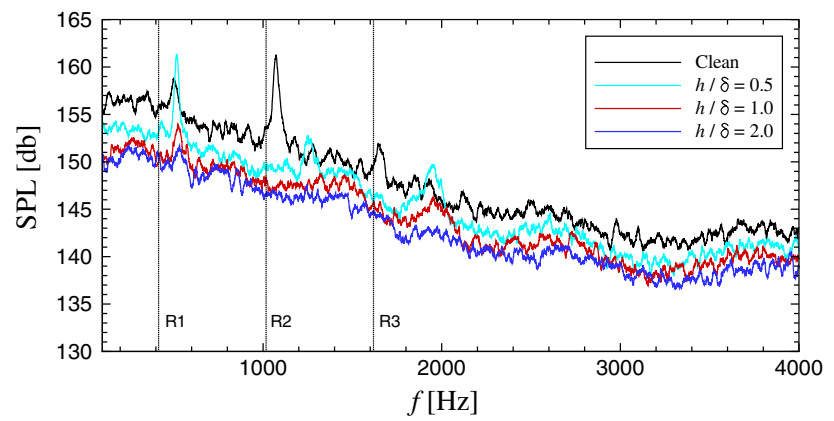

(b) Cavity B.

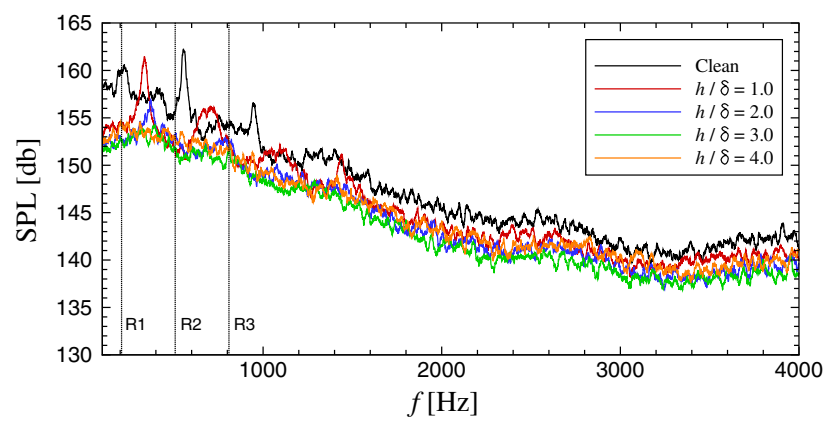

(c) Cavity C.

Fig. 13 The effect of different sawtooth spoiler heights on the sound pressure levels for cavities A, B and C $(x / L=0.9)$

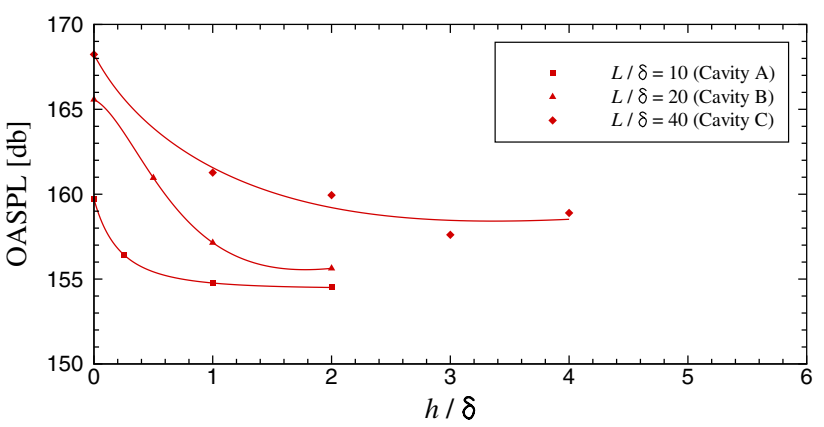

Fig. 14 Variation of OASPL with $h / \delta$ and $L / \delta(x / L=0.9)$

after attenuation of all tones is due to broadband noise. This broadband noise is created mainly due to turbulent fluctuations in the shear layer. With changes in scale or $L / \delta$, the turbulent fluctuations could change. At larger cavity scales, the broadband noise is higher due to the growth and convection of the turbulent fluctuations in the streamwise direction.

The values of OASPL obtained near the aft wall for spoilers of different $h / \delta$ are shown in Fig. 14. It can be seen that the OASPL curves are dependent on the value of $L / \delta$. It was discussed above that a spoiler with height equal to the boundary-layer thickness was insufficient to attenuate the tones for cavity C. However, when the spoiler height was raised to three times the boundary-layer thickness, the tones were completely attenuated to the broadband level (see Fig. 13c). This shows that the spoiler heights have to be adjusted with a change in $L / \delta$. In the literature, the size required for a leading-edge control $\left(h_{\mathrm{cr}}\right.$ here) has been assumed to depend only on factors such as Mach number, $L / D$ and $L / W$. Here, however, the results indicate that the critical spoiler height, $h_{\mathrm{cr}}$, required for a particular cavity is dependent on the factor $L / \delta$, i.e. on the boundary-layer thickness relative to the cavity size. Hence, for a given 


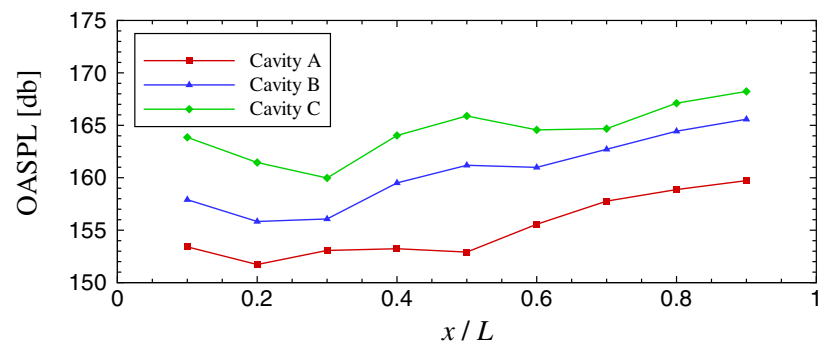

(a)

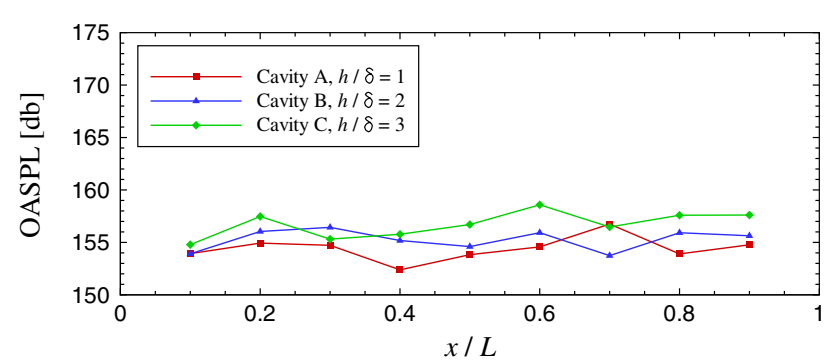

(b)

Fig. 15 The effect of sawtooth spoilers of critical height, $h_{\mathrm{cr}}$, on the sound pressure levels along the floor of the cavities. a Clean configuration. b With sawtooth spoilers employed

cavity, $h_{\mathrm{cr}}=f\left(M_{\infty}, L / D, L / \delta\right)$. The value of $h_{\mathrm{cr}}$ is defined in this work as the lowest value of $h$ that achieves a condition where all the tones are completely eliminated and the OASPL is a minimum, or within $\pm 0.25 \mathrm{~dB}$ of the minimum value. The value of $h_{\mathrm{cr}} / \delta$ for cavities $\mathrm{A}, \mathrm{B}$ and $\mathrm{C}$ obtained from the current experiments can be observed as 1,2 and 3 , respectively, from Fig. 14. It can also be seen from Fig. 14 that increasing the value of $h / \delta$ beyond $h_{\mathrm{cr}} / \delta$ slightly increases the OASPL. This appears to be because a further increase in spoiler height increases the turbulent broadband noise generated by the spoiler.

Figure 15 shows the OASPL variation in the streamwise direction for the three cavities in a clean configuration and using spoilers with critical height, $h_{\mathrm{cr}}$. For the clean cavities (Fig. 15a), it can be seen that the OASPL generally has higher values near the trailing edge. This increase is due to the amplification of the shear-layer disturbances in the downstream direction. For a fixed boundary-layer thickness, the OASPL values increase as the cavity size is increased; again this is due to the greater opportunity for downstream amplification. For the case with spoilers (Fig. 15b), the OASPL for each cavity is reduced to an approximately constant broadband level. The streamwise amplification of the disturbances is effectively suppressed.

The values of $h_{\mathrm{cr}} / \delta$ obtained are plotted against the corresponding values of $L / \delta$ including the point $(0,0)$ in Fig. 16. Uncertainty bars (based on $50 \%$ of $\Delta h$ ) are given for the different values of $h_{\mathrm{cr}} / \delta$ because not all values of $h / \delta$ have been tested. Since $h_{\mathrm{cr}} / \delta$ is chosen as the lowest value of $h / \delta$

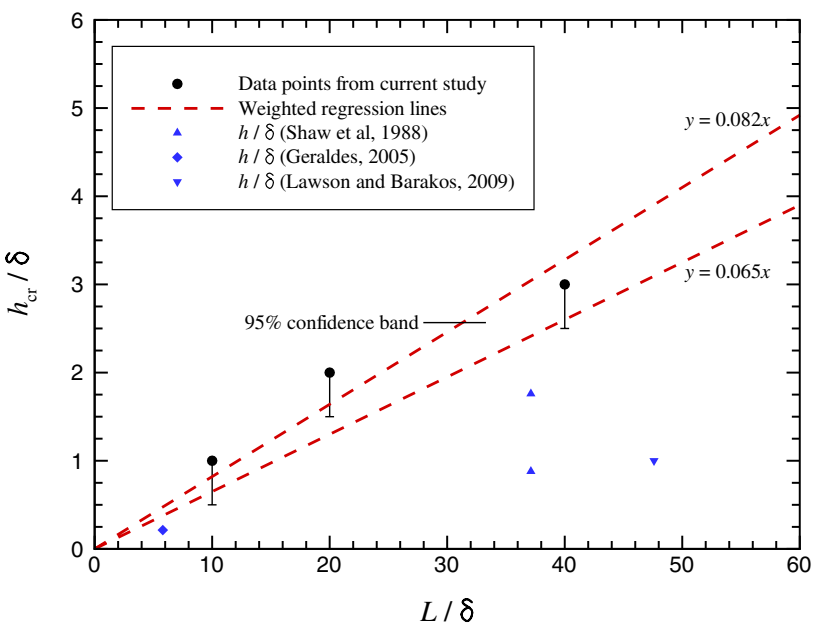

Fig. 16 The linear relationship between $h_{\mathrm{cr}} / \delta$ and $L / \delta$

when all tones are eliminated, only a lower limit is taken. Figure 16 shows the data points obtained with their uncertainties. Using the points available, a linear approximation has been made. A linear regression curve, weighted according to the error at each point, is constructed. Due to the uncertainties, a confidence band (at $95 \%$ confidence level) is also shown.

It can be seen from Fig. 16 that the confidence band is bound within the regression lines $y=0.082 \times$ and $y=0.065 \times$. From this linear approximation, it is clear that the value of $h_{\mathrm{cr}}$ is dependant only on the cavity length $L$ such that

$h_{\mathrm{cr}}=0.065 \leq L \leq 0.082$

This implies that oscillations in a cavity can be suppressed as long as $h / L \geq h_{\mathrm{cr}} / L$. It must be noted that although $h_{\mathrm{cr}} / L$ is independent of the boundary-layer thickness, suppression is not achieved for a given cavity until this ratio is achieved. For $h / L<h_{\mathrm{cr}} / L$, cavity oscillations are still vulnerable to changes in the ratio $L / \delta$. Once $h / L=h_{\mathrm{cr}} / L$ oscillations are suppressed and $L / \delta$ no longer plays a role in cavity unsteadiness.

Previous researchers such as Shaw et al. (1988), Geraldes (2005) and Lawson and Barakos (2009) have presented data on sawtooth spoilers similar to the one used in this work; however, it must be noted that although they observed cavity tone attenuations, Shaw et al. (1988) and Lawson and Barakos (2009) were unable to suppress successfully all the tones to broadband level [see, for example, Figure 11 in Shaw et al. (1988)].

The value of $h / \delta$ used in the works by Shaw et al. (1988) and Lawson and Barakos (2009) is also shown in Fig. 16. Although Shaw et al. (1988) do not specifically quote boundary-layer thickness, there are sufficient data within the report to make an estimate; this has been calculated to 


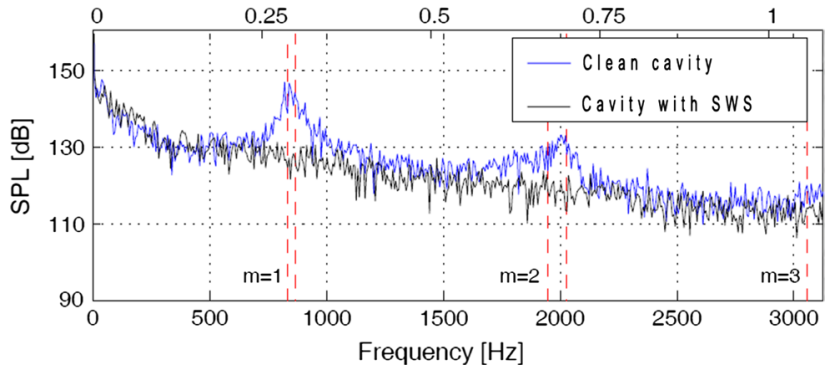

Fig. 17 Cavity noise suppression with sawtooth spoilers (Geraldes 2005)

be $5.2 \mathrm{~mm}$, based upon a flat plate calculation. Referring to an earlier report on the F-111 weapons bay (Clark 1979), the full-scale boundary-layer thickness at the leading edge of the weapons bay was measured as $88.9 \mathrm{~mm}(4.4 \mathrm{~mm}$ at $4.9 \%$ scale) and between 8.0 and $8.4 \mathrm{~mm}$ at $6.67 \%$ scale (5.9-6.2 $\mathrm{mm}$ at $4.9 \%$ scale), giving confidence in the estimated value. It is noted that the value of $h / \delta$ used by these researchers falls below the value of $h_{\mathrm{cr}} / \delta$ recommended by the linear model. By contrast, the value of $h / \delta$ used by Geraldes (2005) is close to the linear approximation, and a good level of suppression was achieved in his studies (Fig. 17). According to the linear approximation model presented here, a cavity with $L / D=5$ achieves suppression when the height of the sawtooth spoiler used is 6.5-8.2\% of the cavity length. This is irrespective of the value of $\mathrm{L} / \delta$ faced by the cavity and indicates that sawtooth spoilers with critical height have to be scaled according to the cavity dimensions and not according to the incoming boundary-layer thickness as previously thought. Once the critical spoiler height, $h_{\mathrm{cr}}$, of a particular cavity is determined, then the spoiler can be scaled according to the cavity dimensions. It is presumed that the critical spoiler height is mainly dependent on factors like spoiler profile and $L / D$ ratio of the cavity. For the current case, a spoiler with height equalling $6.5-8.2 \%$ of the cavity length achieves critical height. A more accurate estimate of $h_{\mathrm{cr}} / L$ can be estimated by testing a large number of the intermediate values in the $h / \delta$-OASPL curves. Shaw et al. (1988) and Lawson and Barakos (2009) quote spoiler heights equalling 4.7 and $3.76 \%$ of the cavity lengths, respectively, which are less than the critical spoiler heights required.

\section{Conclusions}

The influence of scaling on the effectiveness of a sawtooth passive control device for cavity tone suppression was studied. By using spoilers of the same $h / \delta$ ratio on cavities of different scales, it was found that there was significant variation in the control effectiveness. Spoilers with $h / \delta=1$ were able to suppress the tones effectively on smaller cavities, but they failed at larger scales. These results show that scaling the spoiler with respect to the boundary-layer thickness is not correct. It also shows that the factor $L / \delta$ has significant impact on the effectiveness of the passive control.

Different spoiler heights were tested for a given cavity (or $L / \delta$ ), and it was found that the spoilers were effective in eliminating the cavity tones when the ratio of spoiler height to onset boundary-layer thickness, $h / \delta$, reached a particular value. After this value, any further increase in spoiler height was found to have negligible effect. The spoiler height at which this phenomenon was achieved was termed the critical spoiler height, $h_{\mathrm{cr}}$. By correlating the ratios, $h_{\mathrm{cr}} / \delta$ and $L / \delta$, it was found that they are approximately linearly related to each other. The linear model implied that the ratio $h_{\mathrm{cr}} / L$ is constant for a cavity of given $L / D$ and is immune to the effects of $L / \delta$. This implies that as long as $h_{\mathrm{cr}} / L$ is achieved, the cavity is expected to have no tones and exhibit the lowest OASPL possible for that particular $L / \delta$. Once $h_{\mathrm{cr}} / L$ for a particular cavity specification $\left(L / D, L / W, M_{\infty}\right.$ and spoiler profile) is known, then the passive control method can be scaled according to the cavity dimensions. If the spoiler height is less than $h_{\text {cr }}$, then the cavity is not immune to the changes in $L / \delta$. Finally $h_{\mathrm{cr}} / L$ is suggested to be a more suitable criterion for scaling a passive control device because it depends only on the physical cavity dimensions and not the flow conditions.

Open Access This article is distributed under the terms of the Creative Commons Attribution 4.0 International License (http://creativecommons.org/licenses/by/4.0/), which permits unrestricted use, distribution, and reproduction in any medium, provided you give appropriate credit to the original author(s) and the source, provide a link to the Creative Commons license, and indicate if changes were made.

\section{References}

Ashworth R (2008) DES of a cavity with spoiler. In: Peng SH, Haase $\mathrm{W}$ (eds) Advances in hybrid RANS-LES modelling, notes on numerical fluid mechanics, vol 97. Springer, Berlin, pp 162-171

Atvars K, Knowles K, Ritchie SA, Lawson NJ (2009) Experimental and computational investigation of an 'open' transonic cavity flow. Proc Inst Mech Eng G J Aerosp 223(4):357-368

Cattafesta LN, Song Q, Williams DR, Rowley CW, Alvi FS (2008) Active control of flow-induced cavity oscillations. Prog Aerosp Sci 44(7-8):479-502

Christensen K (2004) The influence of peak-locking errors on turbulence statistics computed from PIV ensembles. Exp Fluids 36:484-497

Clark R (1979) Evaluation of F-111 weapon bay aero-acoustic and weapon separation improvement techniques. Technical Report AFFDL-TR-79-3003, Air Force Flight Dynamics Laboratory, Wright Patterson Airforce Base 
Geraldes P (2005) Instabilities in transonic cavity flows. PhD thesis, Cranfield University

Heller H, Holmes D, Covert E (1971) Flow-induced pressure oscillations in shallow cavities. J Sound Vib 18:545-553

Keane RD, Adrian RJ (1992) Theory of cross-correlation analysis of PIV images. Appl Sci Res 49:191-215

Lada C, Kontis K (2011) Experimental studies of open cavity configurations at transonic speeds with flow control. J Aircr 48(2):719-724

Lawson SJ, Barakos GN (2009) Assessment of passive flow control for transonic cavity flow using detached-eddy simulation. J Aircr 46(3):1009-1029

Lusk T, Cattafesta L, Ukeiley L (2012) Leading edge slot blowing on an open cavity in supersonic flow. Exp Fluids 53(1):187-199

MacManus DG, Doran DS (2008) Passive control of transonic cavity flow. J Fluid Eng 130(6):064501-064501-4

Martinez MA, Cicca GMD, Iovieno M, Onorato M (2012) Control of cavity flow oscillations by high frequency forcing. J Fluid Eng 134(5):051201-051201-11

Perng SW, Dolling DS (2001) Suppression of pressure oscillations in high-Mach-number, turbulent, cavity flow. J Aircr 38(2):248-256

Ritchie SA (2005) Non-intrusive measurements and computations of transonic cavity flows with applications to aircraft stores release. $\mathrm{PhD}$ thesis, Cranfield University

Ross J (2001) High speed acoustic measurements in cavities. Technical Report F49620-98-1-0167, DERA
Rossiter JE (1966) Wind-tunnel experiments on the flow over rectangular cavities at subsonic and transonic speeds. Reports and Memoranda 3438, Aeronautical Research Council

Shaw L (1989) Scale effect on the flow induced acoustic environment in cavities. Technical Report WRDC-TM-89-159-FIBG, Wright Research and Development Center, Wright Patterson Airforce Base

Shaw L, Clark R, Talmadge D (1988) F-111 generic weapons bay acoustic environment. J Aircr 25(2):147-153

Thangamani V, Knowles K, Saddington AJ (2014) The effects of scaling on high subsonic cavity flow oscillations and control. J Aircr 51(2):424-433

Ukeiley LS, Ponton MK, Seiner JM, Jansen B (2004) Suppression of pressure loads in cavity flows. AIAA J 42(1):70-79

Vikramaditya NS, Kurian J (2009a) Effect of aft wall slope on cavity pressure oscillations in supersonic flows. Aeronaut $\mathbf{J}$ 113(1143):291-300

Vikramaditya NS, Kurian J (2009b) Pressure oscillations from cavities with ramp. J Aircr 47(12):2974-2984

Westerweel J (1994) Efficient detection of spurious vectors in particle image velocimetry data. Exp Fluids 16:236-247

Westerweel J (1997) Fundamentals of digital particle image velocimetry. Meas Sci Technol 8:1379-1392 